\title{
Cause Placement: Initial Empirical Findings: An Abstract
}

\author{
Ream Shoreibah and Barbara Lafferty
}

\begin{abstract}
The use of embedded marketing, the practice of seamlessly inserting advertising messages into entertainment media, continues to grow as media consumption shifts to on-demand forms and marketers find it increasingly more challenging to reach audiences. Cause placement is the proposed term for the form of embedded marketing used in social and nonprofit marketing; it involves inserting messages about pro-social causes into entertainment programming. Two studies investigated the effects of various message factors on three measures of the effectiveness of cause placement: recall of the cause, attitude toward the cause, and intention to support the cause. The first study examined the effects of brandedness of the cause and placement modality on the measures of cause placement effectiveness, using a 2 (branded/unbranded) by 3 (verbal/visual/both) between-subjects design. A branded cause yielded better recall of the cause and better attitude toward the cause than an unbranded one regardless of placement modality. There were no significant differences among the groups for intention to support the cause, likely due to a ceiling effect. In the second study, recall of the cause was higher when the main character's behavior was inconsistent with his personality, regardless of the image of the character. The second study investigated the effect of image of the character and consistency of the behavior being promoted with the character's personality on the three measures of cause placement effectiveness, using a 2 ("good guy"/"bad guy") by 2 (consistent/inconsistent) between-subjects design. There was an interaction effect between consistency of the behavior and image of the character, such that attitude toward the cause was higher for consistent than inconsistent behavior when the image of the character was "bad guy," but there was no significant difference when the image of the character was "good guy." An analogous pattern for intention to support the cause did not hold, perhaps due to the participants' sense of moral obligation.
\end{abstract}

\author{
R. Shoreibah $(\bowtie)$ \\ Birmingham-Southern College, Birmingham, AL, USA \\ e-mail: rashorei@bsc.edu \\ B. Lafferty \\ University of South Florida, Tampa, FL, USA \\ e-mail: lafferty@usf.edu
}

